\section{ABC presents Nobel Prize Winners in Chemistry}

\author{
Aldo Roda
}

Published online: 2 February 2010

(C) Springer-Verlag 2010

Recently the Editorial board of Analytical and Bioanalytical Chemistry decided to devote, every year, a brief article to highlighting the Nobel Prize laureate(s) in Chemistry. Readers will find two Feature Articles highlighting the winning work in 2008 and 2009 in this journal issue.

The foundation for the establishment of the Nobel Prize was laid in 1895 when Alfred Nobel wrote his last will, leaving much of his wealth to the organization of the Nobel Prize. But, who was Alfred Nobel? Readers may not know that Alfred Nobel was a Swedish chemist, engineer, and the inventor of dynamite. Some believe that he established the Prize to compensate for the "evil" that dynamite may have caused. Whether this is true or not, we do not know, because dynamite is also essential for many important "good" industrial and other applications.

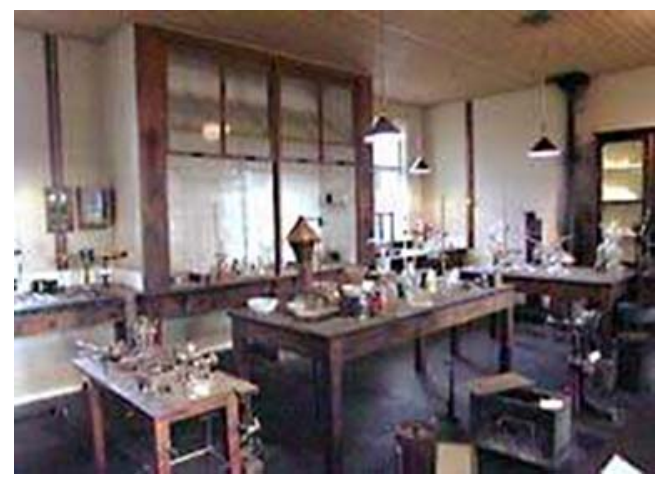

Alfred Nobel's laboratory in Bofors, Sweden. (B) The Nobel Foundation

\section{A. Roda $(\triangle)$}

Laboratory of Analytical and Bioanalytical Chemistry,

Department of Pharmaceutical Sciences,

Alma Mater Studiorum-University of Bologna,

Via Belmeloro 6,

40126 Bologna, Italy

e-mail: aldo.roda@unibo.it
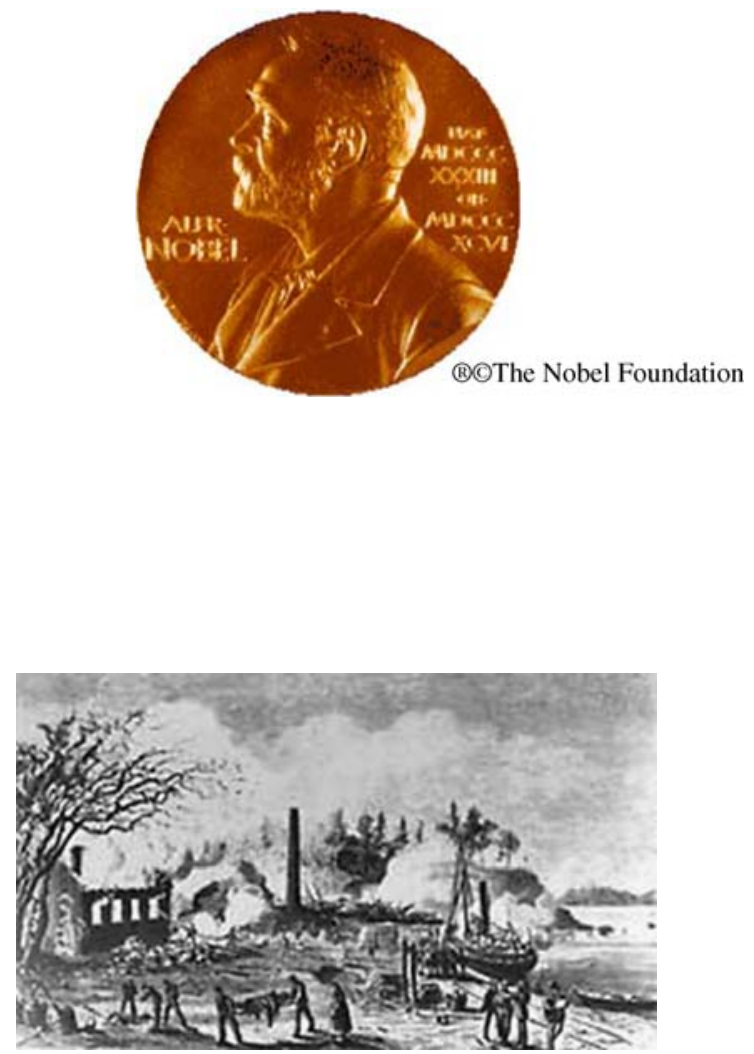

Explosion in the laboratory in Stockholm ()ㅜ The Nobel Foundation

The reasons for launching a Nobel feature into our journal are multiple: The first, and perhaps the most significant, is that the chemistry scientific community is always proud to be able to recognize distinguished colleagues that achieve this prestigious recognition of their research activities. The achievement of this award is an example to "emulate" for scientist of all ages and serves as a stimulus for young researchers to pursue their dreams.

The Nobel Prize recognition of scientists, for example chemists, clearly demonstrates the achievement of important objectives throughout continuous, meticulous, and creative day-by-day work which produced advances in basic chemistry concepts and theory along with technological application to varied fields of chemistry.

The second reason is related to the fact that many recent Nobel Prizes have been awarded not to a single researcher, but rather shared by two or three scientists who did the scientific work independently, reaching a goal by tackling the problem from different angles. Nowadays, the internet and abundant sophisticated multimedia are able to keep the scientific community very close to, and up-to date on, new scientific developments. This also facilitates continuous debate and exchange of information during conferences and 
workshops, allowing for quick advances in science and technology, which in turn, stimulates strong competition among different groups around the world.

The success in the creation of new concepts and their subsequent application stems not only from great ideas, but also from their implementation. The latter is based on a combined systematic approach largely based on grant/ sponsorship funding of the basic ideas and work, followed by technology transfer of this basic work to the private sector, which gambles on a big pay-off resulting from innovation and high-risk investments .

Upon examination of all the Nobel Prizes awarded, from the first in 1901, we can observe how chemistry has evolved in the last hundred years, and, from that, we can classify it into two main periods: First, the period of the pioneers/fathers of modern chemistry, and, second, the time of the main discoveries. Herein, we will discuss selected important achievements that were fundamental to analytical chemistry.

The first period, which spanned the early nineteen hundreds, was very fertile: The first Nobel Prize in Chemistry was awarded in 1901 to Jacobus H. van't Hoff for his discovery of the laws of chemical dynamics and osmotic pressure in solutions. Svante Arrhenius was awarded the Nobel Prize in 1903 for the electrolytic theory of dissociation. Wilhelm Ostwald in 1909 became a laureate for describing the fundamental principles governing chemical equilibria and rates of reaction, and Marie Curie became the first woman to receive the Nobel Prize in 1911 for her services to the advancement of chemistry by discovering the elements radium and polonium, isolating radium, and studying the nature and compounds of radium.

Through the years of World War I, the Nobel Prize was interrupted for just one year; in the peace years thereafter very important new discoveries in chemistry were achieved: Walther Hermann Nernst in 1920 was awarded the Nobel Prize in recognition of his work in thermochemistry, Irving Langmuir in 1932 for his discoveries and investigation of surface chemistry, Harold Clayton Urey in 1934 for his discovery of heavy hydrogen, Frédéric Joliot and Irène Joliot-Curie in recognition of their synthesis of new radioactive elements, and Petrus (Peter) Josephus Wilhelmus Debye in 1936 for his contributions to our knowledge of molecular structure through his research on dipole moments and on the diffraction of X-rays and electrons in gases.

The Nobel Prize Laureates mentioned and, in general, all Laureates set the basics and paved the way for modern chemistry. During the Second World War the awards were partially interrupted between 1940 and 1942 .

The post-World-War period was very productive for analytical chemistry-related discoveries.

In 1943, the Hungarian George de Hevesy was awarded the Nobel Prize for his work on the use of isotopes as tracers in the study of chemical processes. The 1952 Nobel Prize was particularly important, because Archer John Porter Martin and Richard Laurence Millington Synge were recognized for their invention of partition chromatography. Seven years later, in 1959, the laureate was Jaroslav Heyrovsky for discovery and development of polarographic methods of analysis. Other remarkable work applauded by the Nobel Institute in 1960 was that of Willard Frank Libby who developed the method of carbon-14 dating for applications in archaeology, geology, geophysics, and other branches of science. The years 1960-70 were the decade of the macromolecules, polymers, proteins and enzymes, and the decade 1970-80 highlighted the pivotal roles of nucleic acids and biochemical reactions in living organisms. In 1982, Sir Aaron Klug received the Nobel Prize for his development of crystallographic electron microscopy technology and his structural elucidation of biologically important nuclei acid-protein complexes. Research focussing on chemical structure, protein, and nucleic acids continued during the 80 s.

In 1991 Richard R. Ernst was awarded the prize "for his contributions to the development of the methodology of high-resolution nuclear magnetic resonance (NMR) spectroscopy". The 1993 Nobel Prize in Chemistry was especially significant because it highlighted the new era in molecular biology with a critical achievement, that of the invention of the polymerase chain reaction (PCR) method. PCR revolutionised scientific research and discovery not only by allowing better understanding of nature and natural processes but also making possible the development of new DNA-based chemistry, which has found a myriad applications in biomedicine and diagnostics, and in virtually every field of science and engineering. The inventor of PCR, Kary B. Mullis, from La Jolla, California, shared the 1993 Nobel Prize for his invention with Professor Michael Smith of the University of British Columbia, Vancouver, Canada, for his fundamental contributions to the establishment of oligonucleotide-based, site-directed mutagenesis and its development for protein studies. Smith's work was also seminal in building the field of protein design and engineering. There are too many valuable applications of Mullis' PCR method to be able to list them all here, but what we should emphasize is that PCR is, perhaps, the most popular tool employed in molecular biology to date. The beauty of PCR is that it is so simple in concept yet so powerful as an invention. It is, for example, possible to multiply a given DNA segment from a complicated genetic material millions of times in a few hours by using simple equipment. The method constantly offers new possibilities particularly in medical diagnostics, and has been used, for example, to discover the HIV virus or faulty genes in hereditary diseases. Researchers can also reproduce complete DNA sequences/genes from animals that became 
extinct millions of years ago by using the PCR method on fossil material. There is no doubt that PCR has revolutionised analytical chemistry research as we knew it previously by opening up new avenues in bioanalytical research that would have been unthinkable prior to Mullis' ingenuity and discovery.

In more recent years, the Chemistry Nobel Laureates have increased our understanding of chemical processes and their molecular basis, and have also contributed to many of the technological advances that we enjoy today. If we analyze the Nobel Prize laureates of the 21st Century, almost all the Prizes have been shared by 2 or 3 scientists with the exception of 2006 and 2007. The 2000 Nobel Prize in Chemistry was award jointly to Alan J. Heeger of the University of California at Santa Barbara, Alan G. MacDiarmid of the University of Pennsylvania, and Hideki Shirakawa of University of Tsukuba in Japan for the discovery and development of conductive polymers. Conductive plastics are used in, or being developed industrially for, e.g., anti-static substances for photographic film, shields for computer screens against electromagnetic radiation, and for "smart" windows (that can exclude sunlight). In addition, semi-conductive polymers have recently been developed into light-emitting diodes, solar cells, and as displays in mobile telephones and mini-format television screens. Electroanalytical chemists have found these molecules very attractive and have incorporated them in the design and development of new analytical devices and biosensors using doped conducting polymer in which electrons are removed (by oxidation) or introduced (by reduction). A spectacular year for analytical chemists was 2002: The Nobel Prize recognized the efforts of scientists in the development of methods for identification and structural analysis of biological macromolecules. Half of the Prize went to John B. Fenn and Koichi Tanaka for their work on soft desorption ionisation methods for mass spectrometric analyses of biological macromolecules, and the other half to Kurt Wüthrich for the development of nuclear magnetic resonance spectroscopy for elucidation of the threedimensional structure of biological macromolecules in solution. This period was characterized by chemistry applied to cellular events in very complex biological systems.

The year 2007 marked a very import contribution of the Nobel Laureate Gerhard Ertl for his studies of chemical processes on solid surfaces. It should be noted that when examining the history of the scientists who have been distinguished with the Nobel Prize on more than one occasion, we observe that out of the four laureates who have received two Nobel Prizes, three received one of them in chemistry: Maria Skłodowska-Curie: in Physics in 1903, for the discovery of radioactivity, and in Chemistry 1911, for the isolation of pure radium. Linus Pauling: in Chemistry in 1954, for his research into the nature of the chemical bond and its application to the elucidation of the structure of complex substances, and in Peace in 1962, for his relentless nuclear test-ban treaty activism (another piece of trivia is that the colourful Pauling is the only person to have won two unshared Nobel Prizes); Frederick Sanger: in Chemistry in 1958, for elucidation of the structure of the insulin molecule, and in Chemistry in 1980, for understanding viruses by sequencing the virus genetic material, namely its nucleotides.

There is no doubt that the above discoveries and subsequent recognition by the Nobel Institute have positioned Chemistry as one of the more important scientific fields representing the last Century's science and technology. Advances in chemistry have contributed, and continue contributing, to an increase in the quality of life, in finding cures for old and new diseases, in enabling detection, identification, and quantification of many of the major biochemical pathways in organisms and cells, in understanding better materials for industry applications, and in revealing the impact of chemicals in the environment.

We expect future Nobel Prizes to be awarded for important contributions to the field of green chemistry and for new discoveries aimed at conserving and protecting our planet from pollution and destruction of its natural resources. The latter is crucial for ensuring the well-being of future generations, and should be one of our major objectives as chemists and inhabitants of the planet Earth. We can certainly achieve our goal of preserving the environment by emulating the will and determination that other chemists have shown through the years, a few of them, as described above, previous Nobel laureates in Chemistry.

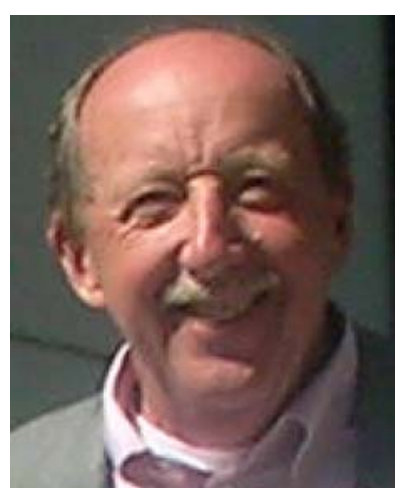

Aldo Roda

is Full Professor of Analytical Chemistry at Bologna University. His main research interests center on the area of analytical and bioanalytical chemistry applied to clinical chemistry, pharmacotoxicology, medicinal chemistry, and environmental and food analysis. He is the owner of more than 20 international patents on new bile acids and antioxidant drugs, new lanthanide chelates as luminescent labels and new luciferases. 